\title{
University Students' Use and Perceptions of Telegram to Promote Effective Educational Interactions: A Qualitative Study
}

\author{
https://doi.org/10.3991/ijet.v16i09.19281 \\ Hibah Khalid Aladsani \\ King Faisal University, Al-Ahsa, Saudi Arabia \\ haladsani@kfu.edu.sa
}

\begin{abstract}
Student interaction is a pivotal element in any educational environment. Accordingly, employing technologies to increase these educational interactions has attracted both instructors' and students' attention. This qualitative paper investigated the means of employing Telegram, a social network site (SNS), to increase students' educational interactions and explore their perceptions of using it as an interactional medium in a university course. A thematic analysis was applied to assess data collected from the posts of 77 university students in three Telegram groups created for this course and the students' reflection papers required at the end of the course. This study's findings identified several instructional activities that can be employed on Telegram to enhance students' interactions, as well as presented how students interact with their instructor and each other on Telegram. The findings also highlighted the students' perceptions of Telegram as a technology to enhance their course interactions, including the advantages and disadvantages of using Telegram in this course. Implications of this study can allow university instructors and policymakers to reconsider their teaching methods and even encourage using Telegram or similar SNSs to aid students' learning.
\end{abstract}

Keywords - Social network sites (SNSs), social media, Telegram, Interaction, Instructional activities, higher education

\section{$1 \quad$ Introduction}

Many studies have demonstrated the importance of interaction between students themselves and between them and their instructors as a significant component to acquiring knowledge and developing successful learning [1], [2]. Additional research has discussed several types of interaction in distance education: for example, learnerinstructor interaction, learner-learner interaction, and learner-content interaction [1]. Hillman et al. [3] also identified the need for an examination of learner-interface interaction for online learning.

These interaction types all occur at different times and frequencies during students' academic experiences. The most frequently occurring interaction in educational settings is the learner-instructor interaction, which is more desirable to students due to 
its instructional direction, support, and motivation to achieve the course objectives [1]. Learner-learner interaction is also an essential part of online learning especially due to peer emotional support giving students a sense of belonging to a connected community [4]. The learner-content interaction occurs when the learner receives new information and processes it in his or her mind with previous knowledge and experience, thereby producing or developing his or her cognitive structure [1]. In online learning, the learner must interact with a communication medium, which Hillman et al. [3] named learner-interface interaction. They considered it the first of any online interactions because students cannot interact with instructors or content before encountering an interface.

The growing use of SNSs has significantly changed the way students learn and interact. There are numerous SNSs such as Facebook, Twitter, Instagram and Telegram. Since its inception in 2013, Telegram has become the most downloaded social media application and seen tremendous user growth, reaching 400 million monthly users [5]. As with other social network sites (SNSs), Telegram is free of charge, has unlimited space for cloud storage, supports media-sharing regardless of file size or type, and can be accessed from multiple devices [6]. Telegram also has special features, such as high security, video calls, chat groups and channels, polls, and bots [5].

Encryption and security are what make Telegram a unique SNS. The app offers multiple security settings that offer users extra privacy. These include hiding user phone numbers from the public, providing two-step verification, controlling who is able to see the "last seen" status, and blocking other users to prevent them from seeing the blocker's messages [7]. Secret chat is another great Telegram feature that protects users from censoring with client-to-client encryption that offers message selfdestruction [8], [7]. Polls also serve as voting tools that can settle disagreements between users or allow them to report their availability for events; these can be anonymous or not. Polls also provide Quiz Mode, which identifies only one correct answer [9].

The common use of SNSs has significantly changed the way students learn and interact [8], [10], [11]. Although the importance of online interaction, there is a dearth of research on employing Telegram as one of SNSs to enhance students' interactions in online educational settings. Using qualitative approach, this study investigates what instructional activities are used in practice to promote students' interaction and how students feel about using Telegram for their interactions.

\section{$2 \quad$ Literature Review}

\subsection{Online communication in learning}

Educational interaction is a vital component in the successful learning process, especially in online environments because of the absence of physical communication between the student themselves and between them and their instructors [12]. Previous studies have discussed the four types of interactions presented by Moore [1] and 
Hillman et al. [3] explaining the benefits of these interaction types on students' learning and providing some suggestions to increase these interactions.

Learner-instructor interaction is the interaction type most discussed in literature. There are various ways instructors can continuously interact with their students to bridge the physical gap in online environments. Interaction can be enhanced by providing students with immediate feedback and using their first names when sending instant messages [13]. Offering emotional support is a great way to increase student interaction and decrease their feeling of isolation [14]. Moreover, the instructor's involvement in the discussion thread with their students encourages students to be more active in online learning activities [13]. Vlachopoulos and Makri [13] also emphasized the positive effect of synchronous classes in maximizing the interaction of students. A good interaction between instructors and students leads to improvements in the latter's academic performance, motivation, satisfaction, and persistence to complete a course [14]. Learner-instructor interaction can produce a sense of academic community that may stop the feeling of isolation that usually appears in online learning environments due to the physical separation between students and their instructor [13].

The second type of educational interaction is learner-learner interaction, which occurs when students communicate and participate in educational activities. Instructors can enhance this interaction by encouraging students to participate in threaded discussion forums and coordinate or lead the teamwork [13]. Besides these activities, students can interact with their peers in their synchronous or asynchronous online classes with or without their instructor in attendance [15]. Yu et al. [15] stated that students involved in frequent peer interaction in online courses acquire a considerable amount of information and improve their academic performance.

Instructors can promote learner-content interaction by providing students with instructional videos to watch, research tasks to do [12], and interactive course content to interact with [13]. When learners interact with content, they retrieve the old information, process the new one, and combine both to create the knowledge [12].

Learner-interface interaction is vital for online learning. As students interact with technology to learn and structure their knowledge, the technology should provide its users ease and satisfaction. However, technical problems in an online system could have a negative impact on students' learning and interaction with technology if these problems are not resolved immediately [12].

\subsection{Telegram in higher education}

With its many features, Telegram can be effectively used for academic purposes. Previous literature has discussed students' attitudes toward the use of Telegram in their courses, its advantages and disadvantages, and its effects on students' academic performance. These studies indicated that students enjoy using Telegram, as it makes their courses more interesting [6], [10], [16], [17], [18], [19], [20], [21]. The students' positive attitudes were mostly due to the advantages granted to them from using Telegram in their learning. One of these advantages is Telegram's seamless network, which lets students access their accounts on any device, meaning they can start 
learning on their mobile phones and continue learning on their laptops, therefore maintaining the information flow from different resources without stopping [8], [22].

Students and instructors appreciate Telegram's media sharing support as well. Telegram supports sharing all file formats up to $1.5 \mathrm{~GB}$ [8]. Instructors can employ this feature in their teaching activities and provide their students multimedia information, such as video clips and images, that improve the students' learning [8]. Al-Abdli [17] indicated that students enjoy using Telegram in a course because of the video clips and images their instructors shared. Xodabande [11] similarly emphasized that sharing video broadcasts on Telegram with his students improved their English pronunciation skills. He also explained that teaching pronunciation is a difficult job for teachers; however, on Telegram, learners can play educational video clips listening to native speakers and learn proper pronunciation on their own time and as many times as they want, which is impossible in traditional offline classroom methods.

Telegram groups are a great tool for building communities, as they can accept up to 200,000 members each. These groups can present publicly to anyone who utilizes Telegram's search box or privately to select users with specific invite links. Many studies have demonstrated that Telegram groups assist students in improving their academic performance and gaining higher course scores for several reasons [8], [16], [19], [21], [23]. First, Telegram groups increase student collaboration and discussions with instructors and peers, which provides students new information [10], [19], [23]. Group discussions also enhance students' understanding of the course content due to their frequent discussions with peers [10]. Moreover, some instructors assign peer assessment tasks over Telegram, which are highly beneficial because students learn from each other and thus expand their knowledge and information [16], [10], [22]. Further, peer assessments in Telegram groups promote students' critical thinking skills with the extra attention that students give to their comments, as these comments are presented in the class's Telegram group and subsequently judged and evaluated by teachers and other students [10]. Quick feedback from instructors in Telegram groups is another contributor to students' improved academic performance [16], [10].

Despite the benefits of Telegram, previous studies have also investigated its drawbacks. Telegram's main downfall is technical issues, such as network failure or lack of internet coverage [16], [19], [22]. Students expressed their annoyance with the internet problems, such as internet disconnections or the lack of internet coverage, which disturb their online learning [22]. Further problem is the high cost of internet subscription [22]. Another drawback is Telegram potentially distracting students from the essential aim of using it in their course, which is learning through other, nontraditional means [16], [22]. Aghajani and Adloo [16] indicated that some students forgot to do their writing task on Telegram because they were distracted by the fun offered on the Internet. This fun could occur when a student opens Telegram to send their assignment but is distracted by other Telegram groups they have joined [22]. Distraction may also happen when peers send irrelevant posts in the Telegram class group that disturb the learning use on the platform [22]. Telegram's ease of use may also increase the workload for both students and teachers, especially if the students expect their teacher to be available for them 24/7 [10], leading to their disappointment 
if the instructor does not reply to their queries immediately. Cheating, addiction to Telegram, and sharing misleading materials are the other disadvantages of this platform [22]. Alizadeh [22] reported that spending a great deal of time on Telegram to do assignments causes students to be addicted. He also explained that some of his students admitted they did not write their assignments themselves, instead they simply used copy and paste from other websites.

\subsection{The educational interactions on telegram}

Previous studies have indicated that Telegram's features support various types of online interaction. For example, it supports all file formats and discussions, and it allows groups to maximize learner-instructor and learner-learner interactions, which consequently enhances students' academic outcomes in their online learning courses [8]. Similarly, Aghajani and Adloo [16], who employed Telegram as a medium for language learning, found that it increases students' interactions with peers in a cooperative environment due to online peer assessments assigned through this SNS, as these assessments have a great role in boosting learner-learner interaction. Habibi et al. [10] indicated that learner-instructor interaction is the most common interaction in online learning that is employed Telegram for several reasons. First, Telegram excellently answers students' need for quick feedback. Furthermore, Telegram as an SNS offers students additional opportunities to interact with their instructors beyond the limited time and place of traditional classrooms [10]. These findings are in line with those of [1], who further claimed that learner-instructor interactions are the most desirable interaction type for instructors, as well as learners.

From this reviewed literature, it seems that most of these studies have focused on the results of experiments that determine Telegram's effectiveness in promoting students' academic outcomes or examine students' attitudes toward Telegram. Though this research ultimately promotes the importance of online interaction, there is a dearth of research on employing Telegram to enhance students' interactions in online educational settings. Additionally, little qualitative research has been done to investigate what instructional activities are used in practice to enhance interaction in general and how students feel about using Telegram for their interactions. The present study addresses these research gaps and reveals how instructors can utilize Telegram as a medium to enhance students' online educational interactions in answering these research questions:

RQ1. Which instructional activities on Telegram are used to enhance educational interactions?

RQ2. How do students perceive Telegram as a platform to support their educational interactions? 


\section{$3 \quad$ Methodology}

\subsection{Research design}

In this study, the researcher examined the ways of employing Telegram to increase students' educational interactions and explored the students' perceptions of using Telegram for their interactions. In this research, the instructor was also the researcher. A qualitative design was applied to gather particularly detailed reports from the students on online educational environments to understand and interpret their experiences [24]. The researcher used two methods: a content analysis of both the Telegram posts and the students' reflection papers that collected their perceptions of using Telegram in the course.

\subsection{Participants and context}

Each of the 77 female students who enrolled in the education technology department's mandatory 14-week computer and society course at King Faisal University (KFU), Saudi Arabia participated in this study. Moreover, While the students were all taking the same course, they were all from three separate classes. All the participants' names were removed from this study for their privacy.

\subsection{Research procedure}

In the first week of the second semester in the academic year (the month that the course started was in January and ended in April 2020), the researcher created three separate Telegram groups based on the day of the students' attended lectures, which were the Tuesday group, Wednesday group, and Thursday group. An invitation link to each group's Telegram chat was sent via email to the students who study the course on that day. The instructor did not provide any training lesson to teach how to use Telegram, as this was considered presumed knowledge. The participants were already familiar with Telegram as a popular SNS.

The researcher employed Telegram as an educational and communication tool. While in class, the students were required to post their answers in their Telegram group to the given tasks. Using Telegram was set as a mandatory course requirement by the instructor, who established certain marks that would contribute to the final course grade for those who engaged in the Telegram activities. Alongside using Telegram as a pedagogical method inside the classroom, the students' posts were displayed with an overhead projector. Telegram was also used as a communication tool outside the classroom. However, according to the university's sudden closure as a precaution against the spread of Covid-19, the instructor taught the students through Telegram for full distance learning, though only for the first lecture after the closure. The next lectures were taught via Blackboard, which is the university's learning management system (LMS), per the university's instructions. 


\subsection{Data collection and analysis}

At the end of the course, the participants were required to submit reflection papers on their perceptions and experiences of using Telegram in this course, particularly explaining how Telegram affected their educational interactions. In total, all 77 students' reflections were analysed. All the Telegram posts from the three groups were analysed as another data source after being collected by the researcher through systematic copying and pasting; Telegram as an SNS could be a great resource for qualitative data that provides the researcher important information that cannot otherwise be found through traditional methods [25]. Both data resources supported and completed each other. The students' reflection papers allowed the researcher to examine their opinions and feelings regarding their experience using Telegram in this course. The Telegram posts, meanwhile, supported the students' reflection data but also allowed the researcher to closely observe how she as the instructor and students used Telegram for educational interactions.

The research data was coded thematically and manually analysed within the framework of the research questions and considering the different types of interactions [26]. For the purpose of this research, the analysis focused on identifying the key themes that emerged from the Telegram posts and the students' reflections papers about using Telegram to interact with their classmates and teacher.

\section{$4 \quad$ Findings and Discussion}

The data analysis identified three main themes related to the employment of Telegram to increase students' interactions. They are (1) instructional activities conveyed over Telegram to enhance students' interactions, (2) learner-learner interactions, and (3) students' perceptions of Telegram as a means to enhance their course interactions.

\subsection{Instructional activities conveyed over telegram to enhance students' interactions}

Interaction is crucial element in any educational environment, as knowledge is constructed in learners' minds through their interactions with others, including instructors and peers [27]. Therefore, it is important for instructors to give special attention to activities that promote students' interactions.

In the current study, several instructional activities were employed in the three Telegram groups to promote course interactions. The first of these was to use Telegram to provide announcements and reminders of the course's academic requirements. On Telegram, the first post, which was sent to all three groups, was the course syllabus, which is considered a course announcement. The instructor also posted announcements regarding the update of lecture times and halls. Further, the instructor frequently posted assignment instructions, reminders, and pending deadlines, as Telegram is a great tool for maintaining the flow of course 
announcements and reminders [16], [28]. In the students' reflections, they reported that they turned on Telegram's notifications to be aware of any update in their course. Turning on mobile phone notifications can assist university students who usually have numerous academic tasks and may face difficulties keeping track of their university assignments. Therefore, Telegram's notifications, which conveyed course reminders and updated timetables, could be an excellent way to inform students which tasks are due to do. Notifications can also assist students in organizing their study life, so they remain current with course updates and are reminded of important study work. Despite the benefit of Telegram notifications as a reminder feature, Habibi et al. [10] warned that this feature may produce more workload for the students.

Alongside announcements, the instructor employed Telegram to motivate the students to be active learners and engage in lecture discussions. In each of the three lectures, the instructor divided the students into four or five groups, then posted questions via Telegram, with the Telegram chat group displayed on an overhead projector. The students were required to discuss their responses in each group, then submit their answers through Telegram on their mobile phones "see Figure 2". In their reflections, the students explained how using the overhead projector to display the Telegram discussions provided them the opportunity to see all the students' replies and compare their answers with others, as well as examine the variety of thoughts and views. This concurs with the SNS use identified by Becker and Bishop [29], who stated that reading others' writing, as presented with an overhead projector, helps students improve their own writing and learning through comparing their work with that in their peers' posts. Moreover, the students explained in their reflections how Telegram was useful for shy students who may feel uncomfortable engaging in traditional discussions in the lecture, as they become more active on Telegram discussions. Other studies have marked Telegram's use in education as making the learning environment more enjoyable and less stressful, which supports students' confidence to be active learners engaging in class activities [16], [18]. Further, the students mentioned that Telegram provided them a space for free speech to present their points of view and allowed them to engage in the class discussions each lecture, which could potentially be difficult or even impossible in the traditional classroom. Indeed, Telegram offers students a sense of confidence to express their ideas and opinions, whereas in face-to-face classes, not all students are able to present their thoughts due to, for instance, embarrassment from speaking publicly [20].

Additionally, the students were required to make teaching aids inside the class as an assignment. As a reward for the students' good work, the instructor took photos of these aids and posted them on the Telegram groups to thank these students, motivate them to do their best, and keep these photos as memories on Telegram. The students replied to these posts on Telegram, expressing their excitement and enjoyment in doing the assignment, as well as seeing the shared photos of their work in their given Telegram group. Sharing students' achievements can create a positive educational environment that motivates students to engage in effective interactions among themselves, as well as with their instructors [30]. 


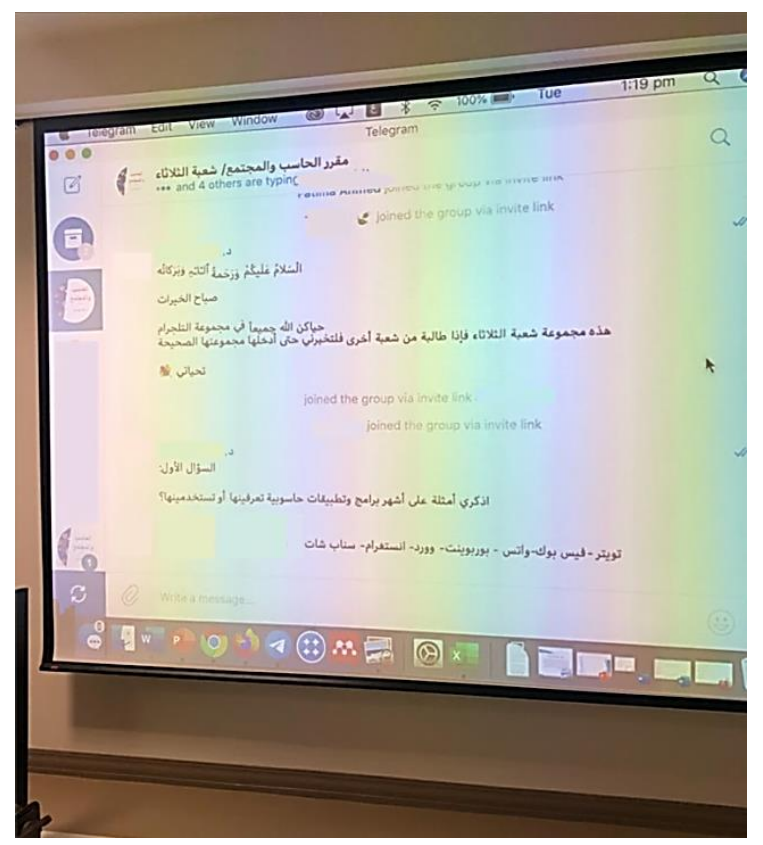

Fig. 1. Using an overhead projector to display the Telegram discussions.

Sharing enriching media, such as video clips and images, was another educational activity on Telegram. In their reflections, the students told that they appreciated these shared media, as they increased their understanding of the lessons' content and thus improved their learning. Their appreciation is similar to that found in [17]. Faramarzi [8] and Xodabande [11], too, emphasized how instructors can support media sharing on Telegram to enhance students' engagement and academic performance.

Telegram's poll feature was also employed to set the most convenient day of the exam for all three groups who studied this course. There were initially arguments between the students regarding this issue, so the instructor asked each one to write the most convenient day for her on the Telegram group. According to these replies, the instructor posted a poll that contained the most frequently proposed days by the students from the three groups. The day that got the highest percentage in the poll was appointed as the exam day.

Inquiries were frequently sent through Telegram to the instructor regarding assignments, exams, grades, and technical issues. In their reflections, the students mentioned that the instructor provided more rapid responses to their questions on Telegram compared to other communication methods, such as email. However, rapid responses from the instructor may lead the students to think that their instructor is available to answer them 24/7, which could increase the instructor's workload [10].

The students also employed Telegram to send their assignments to the instructor; they mentioned some benefits of this format as well in their reflections, such as receiving instant instructor feedback and discussing that feedback with her at the same 
time. Getting fast feedback from teachers is important for students to improve their academic performance [10].

Further, many students wrote that Telegram gave them a sense of friendship and belonging to their instructor that cannot be felt in a traditional classroom. This feeling of friendship could be due to the frequent communication on Telegram that removes the emotional boundaries between students and teachers [10]. Prestridge [31] also stated that communication and interactions on SNSs help students construct a sense of online academic community and strengthen their relationship with their instructor.

\subsection{Learner-learner interaction}

Peer interaction is essential and valuable for online academic environments. It assists students in structuring their knowledge and encourages the development of their expertise and skills [1]. This study's data reported different kinds of learnerlearner interactions, such as social, academic, and emotional interactions.

In the second lecture with Tuesday group, the instructor noticed that there were three students who were engaged in the discussion in their Telegram group, but not attending class inside the classroom. So, the instructor wondered who they were and if they were students from the same group or stranger users who joined the group accidentally. They replied that they were lost and could not find the classroom. However, their classmates inside the class directed them on Telegram to the classroom's location near the stairs. It was a kind of social support between students. The students were happy to use Telegram to provide instant help to their peers. Moreover, this event clearly showed that Telegram is compatible with any device [8]. As a mobile-based app, Telegram can be used immediately, giving it an advantage over computer-based systems. The students offered additional educational assistance to those who were absent and had asked on Telegram about their missed lessons, with the attending students posting summaries of what they were taught in the lecture. The students also assisted each other with technical issues. For example, a student posted on Telegram that she could not hear the instructor's voice on the virtual Blackboard lecture, and one of her classmate told her to try another web browser to join the lecture. Frequent involvement in learner-learner interaction positively affects the academic performance of students [15].

The students also emotionally supported each other. For instance, they encouraged those who were struggling to send assignments by their deadlines and tried to convince each other about the most convenient date for the final exam. In their reflections, the students explained how Telegram gave them a sense of belonging to the other students in their group that they did not feel before in a traditional classroom. The frequent student interactions in online activities increase their emotional closeness beyond the limited time and place during face-to-face sessions [32], [33]. Moreover, educational online interaction on SNSs offer students a sense of academic community with their classmates [29]. In this study, the students further emphasized in their reflections that employing Telegram in the course better encouraged them to become involved in collaborative learning than in traditional lectures. The students explained the benefits of this academic collaboration in 
providing them great prospects to read other students' ideas, experiences, and suggestions, which enhanced their learning. Academic collaboration accompanied by peer comments and feedback on Telegram thus has a pivotal role in improving students' academic performance [16]. Moore [1] emphasized that peer interaction improves the educational skills and expertise of students and sharpens their skills as knowledge creators.

\subsection{Students' perceptions of telegram as a means to enhance their course interactions}

The students presented their perceptions regarding their use of Telegram in this course in their final reflection papers. The researcher noted that talking about the advantages of Telegram as a communication tool was the dominant statements in the students' reflections. Further, the students compared Telegram to other communication methods, as well as discussed, some challenges they faced in using Telegram.

On Telegram, the students expressed their enjoyment with using Telegram as a new technology in their learning. One student posted on Telegram, "Using Telegram in this course is a great opportunity for students in the education technology discipline to apply what we study about educational technology and distance learning". The students stated that as one SNS, Telegram was easy to download and create an account for, as well as enjoyable, exciting [20], free of charge, and compatible with any device or web browser, had great speed and high security [8], and supported Arabic, which was the students' mother tongue, and distance learning.

The students further discussed specific features of Telegram that they utilized. One such feature was saving messages as resource repositories to keep important information that was discussed in the lectures, which offered the students the ability to return to this information to study for the exam, consequently assisting and improving their learning. Telegram's search feature as well was reported as a tool that helped the students find needed information faster inside their course group. The students determined how employing Telegram for class activities could support the natural environment as well by reducing the amount of paper that is used for these activities in traditional settings. The students appreciated Telegram's excellent support for media sharing, regardless of the size or type of media [6]. They also favoured Telegram's synchrony, which allowed them to have conversations with the instructor all at the same time. The students explained how turning on notifications let them be current with the course announcements and not miss anything. The students also mentioned the editing features that helped them edit and improve their answers in the class activities. Telegram's editing feature provides a unique service that is unavailable with a normal delete feature, as it allows the user to edit his or her post to change a character or a word, then repost it without the need to rewrite the entire post. On other SNSs such as Twitter or WhatsApp, when a user wants to correct a word in his or her post after sending it, he or she must delete the first post and rewrite it from the beginning. Thus, Telegram's editing feature saved time and effort, encouraging the students to improve their writing. 
In addition, the students discussed some Telegram features that kept their privacy. For example, private messages meant that they could send their inquiries or assignments to the instructor far away from their peers. Creating a specific group for each class provided them more privacy and was more effective in terms of discussing what was relevant to them, such as a change in lecture time or place. The students also preferred hiding their mobile phone numbers to secure their privacy; although Telegram requires a phone number for registration, its settings offer users the choice to show or hide their phone numbers from the public. In fact, this was one of the reasons the instructor preferred Telegram for this course, as she did not wish to give her personal phone number to the students. Security and encryption made Telegram the top-ranking SNS in terms of high security [5]. Its privacy features include private messages between users, secret chats that offer message self-destruction [8], and the ability to hide user phone numbers from the public [7]. Most of these privacy features are restricted to Telegram only. For example, private messages between users are available on WhatsApp, but the self-destructing messages and phone numbers hidden from the public are unique features of Telegram.

Moreover, the students compared the use of Telegram in their learning to their use of email and Blackboard, which is their university's LMS. The students reported facing challenges in employing Blackboard, such as technical issues, the complexity of practice that needed special training, the need to sign in upon every site access, and delayed media playback. Contrary to these challenges, Telegram was easy to facilitate, constituted a small proportion of technical issues, provided immediate media playback, and automatic sign-in upon use. Faramarzi [8] stated that Telegram is characterized by its the speed of uploading and downloading files due to its structure as a cloud-based system. The students also commended Telegram's advantages over email in some respects, such as supporting collaborative discussion among students, confirming message receipt, and minimizing spelling errors in email addresses. However, regarding message delivery and receipt confirmation, the students were not aware of the delivery receipt feature in email that confirms a message was read by the recipient. One student even complained that she got a zero on an assignment because the instructor did not receive her message through email, and she could not find a way to confirm whether she did or did not read her email. She reported this event by preferring Telegram over email because Telegram provides users with receipt confirmation as she stated.

The students also listed some of the challenges of using Telegram in the course. First was the inability to utilize Telegram in offline mode. Second, Telegram rapidly consumes battery life and cellular data. However, these challenges may appear in most SNS apps that need Internet connection and battery life to operate. Additionally, Telegram does not offer the ability to select more than one file to share, unlike other SNSs such as WhatsApp. Anonymous users on Telegram also frightened some of the students due to the potential harm they can cause. The anonymity is made possible by the feature that hides users' phone number, which was previously considered an advantage by some students. Using Telegram inside the class may additionally distract students, as they can easily browse other sites without their instructor knowing. Several studies supported this finding, explaining how browsing irrelevant 
websites, other Telegram groups that the student has joined, or friend's posts may distract the student far away from class learning [16], [22]. Students also complained that Telegram does not support virtual classrooms.

Despite these challenges, the students' main perception was that Telegram was very useful and valuable to increasing their educational interactions in this course. They enjoyed the educational interactions between themselves and between them and their instructor on Telegram.

\section{$5 \quad$ Conclusion and Implications}

This study sheds light on Telegram as a medium to encourage students' educational interactions. The study's findings present a number of instructional activities that can be employed on Telegram by instructors who perceive interaction as an important aspect of successful learning. Notably, Telegram can be utilized as an announcement and reminder tool to keep students current with course updates. Instructors can also employ Telegram as an exciting medium to motivate students to engage in lecture discussions. Using an overhead projector to display Telegram discussions in front of the class can improve the students' answers to assignments, as they will be seen and appraised by their peers, and motivate shy students to become active in these discussions. Telegram's features also offer various services that support the educational process, such as groups, media sharing, and polls.

With these features, using Telegram can enhance course interactions in general. The educational interactions on Telegram in this particular course were not restricted to learner-instructor interactions, but also involved learner-learner interactions that appeared in several ways, such as social, academic, and emotional interactions between students. In fact, the frequent interactions on Telegram between the students and their instructor and among themselves created a sense of academic community with the instructor and students. The findings also show that the students held positive perceptions of using Telegram in this course, as the students discussed some of Telegram's advantages, such as its ease of use, media sharing support, and strong privacy options. The students ultimately found that Telegram was more useful for interaction than email and Blackboard. They also mentioned some of the challenges they faced while using Telegram. Thus, the challenges the students faced need to be kept in mind should Telegram be implemented in other classrooms or universities.

The study presents some limitations, however. This study examined students' interactions and perceptions only and did not include learning outcomes. It is important to mention that learning content was not addressed in detail here either due to not conducting tests to examine the learners' engagement with online learning content. Future studies can address this issue by using either qualitative, quantitative, or mixed methods. Further studies can also compare the use of Telegram and an official LMS, such as Blackboard, in terms of enhancing students' educational interactions. Another study could address if the course's topic or format (e.g., a large class reliant on instructor lectures vs. a small seminar) influence the results. 
This study's findings may supplement important contributions to our knowledge of Telegram's use to enhance students' educational interactions. University policymakers and instructors can benefit from this study to understand how students perceive Telegram, a popular SNS for social purposes, in educational settings. Additionally, they can learn how to encourage students to engage more in academic interactions by using Telegram as a new and enjoyable medium.

\section{Acknowledgements}

The author acknowledges the Deanship of Scientific Research at King Faisal University for the financial support under Nasher Track (Grant No. 206116)

My thanks to my students for their participation in the study.

\section{$7 \quad$ References}

[1] M. G. Moore, "Editorial: Three Types of Interaction," American Journal of Distance Education, vol. 3, no. 2, pp. 1- 7, 1989.

[2] R. M. Palloff, and K. Pratt, Building learning communities in cyberspace. San Francisco: Jossey-Bass, 1999.

[3] D. C. Hillman, D. J. Willis, and C. N. Gunawardena, "Learner-interface interaction in distance education: An extension of contemporary models and strategies for practitioners," The American Journal of Distance Education, vol. 8, no. 2, pp. 30- 43, 1994. https://doi.org/10.1080/08923649409526853

[4] M. Ghaffari, S. Rakhshanderou, Y. Mehrabi, and A. Tizvir, "Using Social Network of TELEGRAM for Education on Continued Breastfeeding and Complementary Feeding of Children among Mothers: A Successful Experience from Iran," International Journal of Pediatrics-Mashhad, vol. 5, no. 7, pp. 5275- 5286, 2017.

[5] P. Durov, "400 million users, 20,000 stickers, quizzes 2.0 and $€ 400 \mathrm{~K}$ for creators of educational tests," Telegram.org, April. 3, 2020. [Online]. Available: https://telegram.org/blog/400-million. [Accessed: May. 1, 2020].

[6] F. Alkhezzi and W. Al-Dousari, "The Impact of Mobile Learning on ESP Learners' Performance," The Journal of Educators Online, vol. 13, no. 2, pp. 73- 102, 2016. https://doi.org/10.9743/jeo.2016.2.4

[7] E. Vaziripour, R. Farahbakhsh, M. O'Neill, J. Wu, K. Seamons, and D. Zappala, "A Survey of the Privacy Preferences and Practices of Iranian Users of Telegram," in USEC Workshop, 2018, no. February, pp. 1- 20. https://doi.org/10.14722/usec.2018.23033

[8] S. Faramarzi, H. Tabrizi, and A. Chalak, "Telegram: An instant messaging application to assist distance language learning (App Review)," Teaching English with Technology, vol. 19, no. 1, pp. 132-147, 2019.

[9] The Telegram Team, "Polls 2.0: Visible votes, multiple answers, and quiz mode," Telegram.org, January. 23, 2020. [Online]. Available: https://telegram.org/blog/polls-2-0vmq. [Accessed: May. 1, 2020].

[10] A. Habibi, A. Mukminin, Y. Riyanto, L. D. Prasojo, U. Sulistiyo, M. Sofwan, and F. Saudagar, "Building an online community: Student teachers' perceptions on the advantages of using social networking services in a teacher education program," Turkish 
Online Journal of Distance Education, vol. 19, no. 1, pp. 46- 61, 2018. https://doi.org/ $10.17718 /$ tojde. 382663

[11] I. Xodabande, "The effectiveness of social media network Telegram in teaching English language pronunciation to Iranian EFL learners," Cogent Education, vol. 4, no. 1, pp. 114, 2017. https://doi.org/10.1080/2331186x.2017.1347081

[12] G. van den BERG, "Context Matters: Student Experiences of Interaction in Open Distance Learning," Turkish Online Journal of Distance Education, vol. 21, no. 4, pp. 223-233, 2020. https://doi.org/10.17718/tojde.803411

[13] D. Vlachopoulos and A. Makri, "Online communication and interaction in distance higher education: A framework study of good practice," International Review of Education, vol. 65, no. 4, pp. 605-632, 2019. https://doi.org/10.1007/s11159-019-09792-3

[14] F. Froment, J. González, and M. Bohórquez, "The Use of Social Networks as a Communication Tool between Teachers and Students: A Literature Review," TOJET: The Turkish Online Journal of Educational Technology, vol. 16, no. 4, pp. 126-144, 2017.

[15] J. Yu, C. Huang, Z. Han, T. He, and M. Li, "Investigating the influence of interaction on learning persistence in online settings: Moderation or mediation of academic emotions?," International Journal of Environmental Research and Public Health, vol. 17, no. 7, pp. 121, 2020. https://doi.org/10.3390/ijerph17072320

[16] M. Aghajani and M. Adloo, "The Effect of Online Cooperative Learning on Students' Writing Skills and Attitudes through Telegram Application," International Journal of Instruction, vol. 11, no. 3, pp. 433- 448, 2018. https://doi.org/10.12973/iji.2018.11330a

[17] H. Al-Abdli, "The Impact of Teaching by Using Interactive Mobile Applications on the Attitudes of Students, Faculty of Education, Hodeida University, towards the Technology of Education and its Applications," Journal of Educational and Psychological Sciences University of Bahrain, vol. 19, no. 1, pp. 581- 612, 2018. https://doi.org/10.12785/ jeps $/ 190117$

[18] S. Bakar, F. Fauzi, N. Yasin, and M. Yunus, "Compound Chunk: Telegram Autobot Quiz to Improve Spelling on Compound Nouns," International Journal of Academic Research in Progressive Education and Development, vol. 8, no. 1, pp. 48- 63, 2018. https://doi.org/10.6007/ijarped/v8-i1/5509

[19] M. Ibrahim, E. Norsaal, M. Abdullah, Z. Soh, and A. Othman, "Preliminary Perception of Teaching and Learning Using Telegram Social Media Tool," Journal Intelek, vol. 12, no. 2, pp. 95- 103, 2016.

[20] Z. H. Iksan and S. M. Saufian, "Mobile Learning: Innovation in Teaching and Learning Using Telegram," International Journal of Pedagogy and Teacher Education, vol. 1, no. 1, pp. 19- 26, 2017. https://doi.org/10.20961/ijpte.v1i1.5120

[21] Z. Khodarahmi and M. Heidari-Shahreza, "Effect of MALL on the Acquisition of Word Stress Patterns of English by Iranian EFL Learners: The Case of Telegram," Journal of Applied Linguistics and Language Research, vol. 5, no. 1, pp. 40- 55, 2018.

[22] I. Alizadeh, "Evaluating the educational usability of Telegram as an SNS in ESAP programs from medical students' perspective," Education and Information Technologies, vol. 23, no. 6, pp. 2569- 2585, 2018. https://doi.org/10.1007/s10639-018-9731-5

[23] S. Mehri and S. Izadpanah, "The Effect of Computer-mediated Communication Tools in Online Setting on Iranian EFL Learners' Teaching, Social and Cognitive Existence," Journal of Language Teaching and Research, vol. 8, no. 5, p. 978- 985, 2017. https://doi.org/10.17507/jltr.0805.20

[24] J. W. Creswell and C. N. Poth, Qualitative inquiry and research design: Choosing among five approaches, 4th ed., London: Sage Publications Ltd, 2018. 
[25] T. Paulus, J. Lester, and P. Dempster, Digital tools for qualitative research. London: SAGE Publications Ltd, 2014

[26] V. Braun and V. Clarke, "Using thematic analysis in psychology," Qualitative Research in Psychology, vol. 3, no. 2, pp. 77- 101, 2006. https://doi.org/10.1191/1478088706qp063oa

[27] L. Vygotsky, Mind in society. Harvard: Harvard University Press, 1978.

[28] M. Macià and I. García, "Professional development of teachers acting as bridges in online social networks," Research in Learning Technology, vol. 26, pp. 1- 21, 2018. https://doi.org/10.25304/rlt.v26.2057

[29] R. Becker and P. Bishop, “"Think bigger about science ': Using Twitter for learning in the middle grades," Middle School Journal, vol. 47, no. 3, pp. 4-16, 2016. https://doi.org/ 10.1080/00940771.2016.1135097

[30] S. Shan, C. Li, J. Shi, L. Wang, and H. Cai, "Impact of Effective Communication, Achievement Sharing and Positive Classroom Environments on Learning Performance," Systems Research and Behavioural Science, vol. 31, no. 3, pp. 471- 482, 2014. https://doi.org/10.1002/sres.2285

[31] S. Prestridge, "A focus on students' use of Twitter - their interactions with each other, content and interface," Active Learning in Higher Education, vol. 15, no. 2, pp. 101- 115, 2014. https://doi.org/10.1177/1469787414527394

[32] J. Lee and C. J. Bonk, "Social network analysis of peer relationships and online interactions in a blended class using blogs," Internet and Higher Education, vol. 28, pp. 35 44, 2016. https://doi.org/10.1016/j.iheduc.2015.09.001

[33] M. Almasi and C. Zhu, "Students' perceptions of social presence in blended learning courses in a Tanzanian medical college," International Journal of Emerging Technologies in Learning (iJET), vol. 13, no. 9, pp. 107- 122, 2018. https://doi.org/10.3991/ijet. $\underline{\mathrm{v} 13 \mathrm{i} 09.8566}$

\section{Author}

Hibah Khalid Aladsani received her Ph.D. degrees in educational technology from the University of Sheffield, UK, in 2019. Since 2019, she has been an Assistant Professor with the Department of Curriculum and Instruction (Educational Technology), at King Faisal University in Saudi Arabia. Her research interests focus on online learning, educational use of social network sites, and employing new technology in education using qualitative research approach. She acts as a reviewer for various prestigious journals and publishers. She likes tweeting on her account @ HibahKA regarding teaching and researching issues.

Article submitted 2020-10-17. Resubmitted 2020-12-22. Final acceptance 2020-12-23. Final version published as submitted by the authors. 